\title{
Early detection of pancreatic cancer in type 2 diabetes mellitus patients based on ${ }^{1} \mathrm{H}$ NMR metabolomics
}

\author{
Lenka Michálková ${ }^{1,2}$, Štěpán Horník ${ }^{1,2}$, Jan Sýkora ${ }^{1, *}$, Lucie Habartová ${ }^{2}$, Vladimír Setnička $^{2}$ \\ and Bohuš Bunganič ${ }^{3, *}$ \\ ${ }^{I}$ Department of Analytical Chemistry, Institute of Chemical Process Fundamentals of the CAS, Prague \\ 6, Czech Republic \\ ${ }^{2}$ Department of Analytical Chemistry, University of Chemistry and Technology Prague, Prague 6, Czech \\ Republic \\ ${ }^{3}$ Department of Internal Medicine, $1^{\text {st }}$ Faculty of Medicine of Charles University and Military University \\ Hospital, Prague 6, Czech Republic
}

\section{A table of contents:}

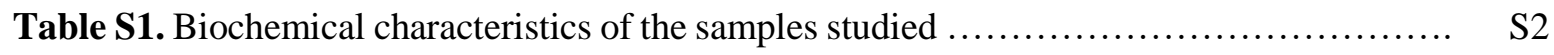

Table S2. A list of acute and chronic medication of PC patients ......................... S4

Table S3. A list of acute and chronic medication of T2DM patients ...................... S5

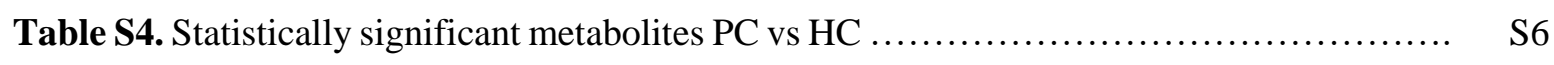

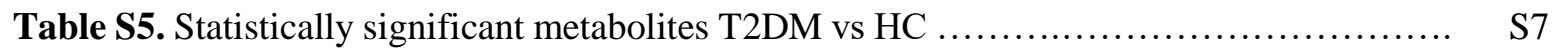

Table S6. Statistically significant metabolites PC vs T2DM $\ldots \ldots \ldots \ldots \ldots \ldots \ldots \ldots \ldots \ldots \ldots \ldots \ldots \ldots \ldots \ldots \ldots \ldots \ldots \ldots$

Table S7. Confusion matrix for simultaneous discrimination of PC, T2DM and HC .......... S7

Figure S1. Metabolic pathway analysis of the group discrimination $\ldots \ldots \ldots \ldots \ldots \ldots \ldots \ldots \ldots \ldots \ldots \ldots \ldots \ldots$

Figure S2. Principal component analyses of group differences based on concentration data .... S9

Figure S3. OPLS-DA based on binning data ..................................... S10

Figure S4. Box plots of dysregulated metabolites between PC, T2DM and HC .............. S11

Figure S5. ROC curves using all 58 metabolites and for specific 8 -metabolite panel .......... S12

Figure S6. CT and MR of RODM 008 patient ...................................... S13

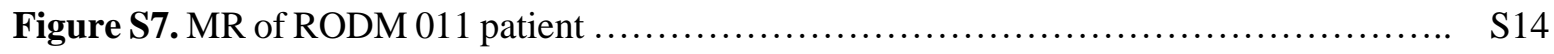

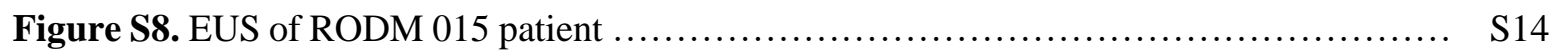

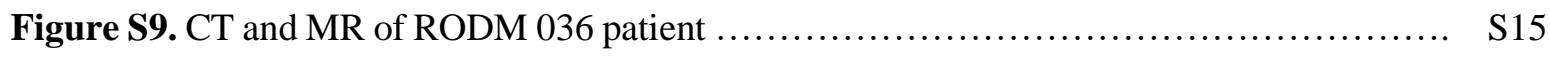

Figure S10. MR and CT of RODM 071 patient ................................ S15 


\section{Biochemical characteristics of the samples}

Table S1. Biochemical characteristics of the samples studied. Data are reported as mean \pm standard deviation, or count.

\begin{tabular}{|c|c|c|c|c|c|}
\hline & & $\mathrm{PC}(n=41)$ & $\mathrm{HC}(n=28)$ & $\mathrm{T} 2 \mathrm{DM}(n=31)$ & $\operatorname{RODM}(n=59)$ \\
\hline Age & & $67 \pm 9.0$ & $64 \pm 9.7$ & $70 \pm 8.6$ & $65 \pm 8.24$ \\
\hline Female & & 16 & 19 & 16 & 32 \\
\hline Male & & 24 & 9 & 15 & 26 \\
\hline Urea & $\mathrm{mmol} \cdot \mathrm{L}^{-1}$ & $4.88 \pm 1.46$ & $4.88 \pm 1.32$ & $5.87 \pm 2.13$ & $5.75 \pm 2.04$ \\
\hline Creatinine & $\mu \mathrm{mol} \cdot \mathrm{L}^{-1}$ & $69.08 \pm 16.96$ & $72.38 \pm 18.10$ & $82.73 \pm 19.09$ & $76.15 \pm 16.87$ \\
\hline Uric acid & $\mu \mathrm{mol} \cdot \mathrm{L}^{-1}$ & $269.88 \pm 92.35$ & $290.37 \pm 66.29$ & $334.90 \pm 98.39$ & $331.60 \pm 89.15$ \\
\hline Sodium & $\mathrm{mmol} \cdot \mathrm{L}^{-1}$ & $139.41 \pm 2.89$ & $141.64 \pm 2.03$ & $140.92 \pm 1.86$ & $140.82 \pm 3.11$ \\
\hline Potassium & $\mathrm{mmol} \cdot \mathrm{L}^{-1}$ & $4.26 \pm 0.49$ & $4.46 \pm 0.39$ & $4.68 \pm 0.48$ & $4.45 \pm 0.44$ \\
\hline Chlorides & $\mathrm{mmol} \cdot \mathrm{L}^{-1}$ & $100.23 \pm 3.46$ & $102.68 \pm 2.15$ & $101.35 \pm 3.73$ & $101.53 \pm 3.27$ \\
\hline Calcium total & $\mathrm{mmol} \cdot \mathrm{L}^{-1}$ & $2.35 \pm 0.21$ & $2.41 \pm 0.10$ & $2.45 \pm 0.15$ & $2.42 \pm 0.11$ \\
\hline $\begin{array}{l}\text { Calcium } \\
\text { ionized }\end{array}$ & $\mathrm{mmol} \cdot \mathrm{L}^{-1}$ & $1.52 \pm 1.66$ & $1.23 \pm 0.05$ & $1.23 \pm 0.04$ & $1.23 \pm 0.05$ \\
\hline Phosphorus & $\mathrm{mmol} \cdot \mathrm{L}^{-1}$ & $1.17 \pm 0.20$ & $1.13 \pm 0.16$ & $1.14 \pm 0.18$ & $1.11 \pm 0.16$ \\
\hline Magnesium & $\mathrm{mmol} \cdot \mathrm{L}^{-1}$ & $0.82 \pm 0.11$ & $0.86 \pm 0.07$ & $0.83 \pm 0.09$ & $0.84 \pm 0.08$ \\
\hline Bilirubin total & $\mu \mathrm{mol} \cdot \mathrm{L}^{-1}$ & $47.24 \pm 64.79^{*}$ & $10.54 \pm 4.37$ & $10.03 \pm 5.05$ & $10.59 \pm 6.62$ \\
\hline Bilirubin direct & $\mu \mathrm{mol} \cdot \mathrm{L}^{-1}$ & $39.01 \pm 58.82 *$ & $4.20 \pm 1.49$ & $4.33 \pm 2.09$ & $4.49 \pm 2.36$ \\
\hline ALT & $\mu \mathrm{kat} \cdot \mathrm{L}^{-1}$ & $3.46 \pm 11.63$ & $0.47 \pm 0.29$ & $0.58 \pm 0.35$ & $0.54 \pm 0.37$ \\
\hline AST & $\mu \mathrm{kat} \cdot \mathrm{L}^{-1}$ & $1.44 \pm 3.67$ & $0.41 \pm 0.18$ & $0.48 \pm 0.26$ & $0.43 \pm 0.21$ \\
\hline GMT & $\mu \mathrm{kat} \cdot \mathrm{L}^{-1}$ & $6.03 \pm 9.04$ & $0.75 \pm 1.53$ & $1.68 \pm 2.67$ & $0.92 \pm 1.42$ \\
\hline ALP & $\mu \mathrm{kat} \cdot \mathrm{L}^{-1}$ & $5.06 \pm 6.53 *$ & $1.48 \pm 1.06$ & $1.40 \pm 0.62$ & $1.59 \pm 1.52$ \\
\hline Amylase total & $\mu \mathrm{kat} \cdot \mathrm{L}^{-1}$ & $0.75 \pm 0.56$ & $1.60 \pm 2.69$ & $1.12 \pm 0.62$ & $0.97 \pm 0.48$ \\
\hline $\begin{array}{l}\text { Pancreatic } \\
\text { amylase }\end{array}$ & $\mu \mathrm{kat} \cdot \mathrm{L}^{-1}$ & $0.37 \pm 0.54$ & $0.93 \pm 2.55$ & $0.60 \pm 0.42$ & $0.42 \pm 0.26$ \\
\hline $\begin{array}{l}\text { Total } \\
\text { cholesterol }\end{array}$ & $\mathrm{mmol} \cdot \mathrm{L}^{-1}$ & $4.80 \pm 1.76$ & $5.57 \pm 0.93$ & $4.26 \pm 1.16$ & $4.95 \pm 1.19$ \\
\hline LDL & $\mathrm{mmol} \cdot \mathrm{L}^{-1}$ & $2.73 \pm 1.29$ & $3.26 \pm 1.02$ & $2.93 \pm 3.88$ & $2.73 \pm 1.02$ \\
\hline $\mathrm{HDL}$ & $\mathrm{mmol} \cdot \mathrm{L}^{-1}$ & $0.98 \pm 0.46$ & $1.96 \pm 1.32$ & $1.30 \pm 0.35$ & $1.37 \pm 0.54$ \\
\hline Triglycerides & $\mathrm{mmol} \cdot \mathrm{L}^{-1}$ & $1.44 \pm 0.57$ & $1.27 \pm 0.52$ & $1.60 \pm 0.90$ & $1.79 \pm 1.10$ \\
\hline FPG & $\mathrm{mmol} \cdot \mathrm{L}^{-1}$ & $6.53 \pm 2.49$ & $5.44 \pm 0.52$ & $7.85 \pm 1.83$ & $7.10 \pm 2.57$ \\
\hline C-peptide & $\mathrm{pmol} \cdot \mathrm{L}^{-1}$ & $653.34 \pm 484.13$ & $\begin{array}{c}710.45 \pm \\
325.94\end{array}$ & $\begin{array}{c}1106.25 \pm \\
342.31\end{array}$ & $\begin{array}{c}1023.92 \pm \\
591.36\end{array}$ \\
\hline Albumin & $g \cdot L^{-1}$ & $37.82 \pm 5.73$ & $45.44 \pm 3.80$ & $45.45 \pm 4.50$ & $44.47 \pm 4.34$ \\
\hline Total protein & $\mathrm{g} \cdot \mathrm{L}^{-1}$ & $63.60 \pm 7.58$ & $70.04 \pm 5.92$ & $73.03 \pm 6.24$ & $69.49 \pm 6.09$ \\
\hline Prealbumin & $\mathrm{g} \cdot \mathrm{L}^{-1}$ & $0.18 \pm 0.08$ & $0.27 \pm 0.07$ & $0.31 \pm 0.08$ & $0.28 \pm 0.08$ \\
\hline
\end{tabular}




\begin{tabular}{llcccc} 
CRP & $\mathrm{mg} \cdot \mathrm{L}^{-1}$ & $22.35 \pm 25.20$ & $2.93 \pm 4.05$ & $19.92 \pm 79.33$ & $7.39 \pm 17.35$ \\
CA 19-9 & $\mathrm{kU} \cdot \mathrm{L}^{-1}$ & $4678.80 \pm$ & $9.99 \pm 5.45$ & $18.57 \pm 13.80$ & $15.26 \pm 11.73$ \\
CEA & $\mathrm{kU} \cdot \mathrm{L}^{-1}$ & $28.02 \pm 65.14$ & $2.29 \pm 1.56$ & $2.18 \pm 1.39$ & $2.32 \pm 2.11$ \\
HbA1c & $\mathrm{mmol} \cdot \mathrm{L}^{-1}$ & $50.83 \pm 21.58$ & $36.22 \pm 3.59$ & $53.69 \pm 15.26$ & $49.37 \pm 17.26$ \\
Leukocytes & $10^{9} \cdot \mathrm{L}^{-1}$ & $7.99 \pm 2.45$ & $6.52 \pm 2.09$ & $7.65 \pm 2.03$ & $7.32 \pm 2.18$ \\
Erythrocytes & $10^{12} \cdot \mathrm{L}^{-1}$ & $5.04 \pm 4.54$ & $4.73 \pm 0.43$ & $4.92 \pm 0.62$ & $4.86 \pm 0.51$ \\
Haemoglobin & $\mathrm{g} \cdot \mathrm{L}^{-1}$ & $124.97 \pm 18.16$ & $141.89 \pm 14.25$ & $145.77 \pm 17.06$ & $143.40 \pm 15.51$ \\
Haematocrit & $\mathrm{g} \cdot \mathrm{L}^{-1}$ & $1.20 \pm 5.04$ & $0.42 \pm 0.04$ & $0.43 \pm 0.05$ & $0.42 \pm 0.04$ \\
Thrombocytes & $10^{9} \cdot \mathrm{L}^{-1}$ & $232.73 \pm 79.18$ & $239.44 \pm 56.64$ & $214.58 \pm 58.92$ & $229.33 \pm 79.24$ \\
INR & & $1.10 \pm 0.19$ & $0.99 \pm 0.05$ & $1.05 \pm 0.11$ & $0.97 \pm 0.15$ \\
Aptt & & $28.25 \pm 7.21$ & $27.36 \pm 5.53$ & $25.62 \pm 6.68$ & $25.57 \pm 8.34$ \\
\hline
\end{tabular}

* Blood was collected before ERCP drain(age), which caused hyperbilirubinemia. (Median of total bilirubin $15.8 \mu \mathrm{mol} \cdot \mathrm{L}^{-1}$, median of direct bilirubin $7 \mu \mathrm{mol} \cdot \mathrm{L}^{-1}$, ALP median $2.83 \mu \mathrm{kat} \cdot \mathrm{L}^{-1}$.)

ALP, alkaline phosphatase; ALT, alanine transaminase; Aptt, activated partial thromboplastin time; AST, aspartate transaminase; CA 19-9, carbohydrate antigen 19-9; CEA, carcinoembryonic antigen; CRP, C-reactive protein; ERCP, endoscopic retrograde cholandiopancreatography; FPG, fasting plasma glucose; GMT, gamma-glutamyl transferase; $\mathrm{HBA}_{1 \mathrm{lc}}$, glycated haemoglobin; HDL, high density lipoproteins; INR, international normalised ratio; LDL, low density lipoproteins. 


\section{Medication}

Table S2. A list of acute and chronic medication of PC patients.

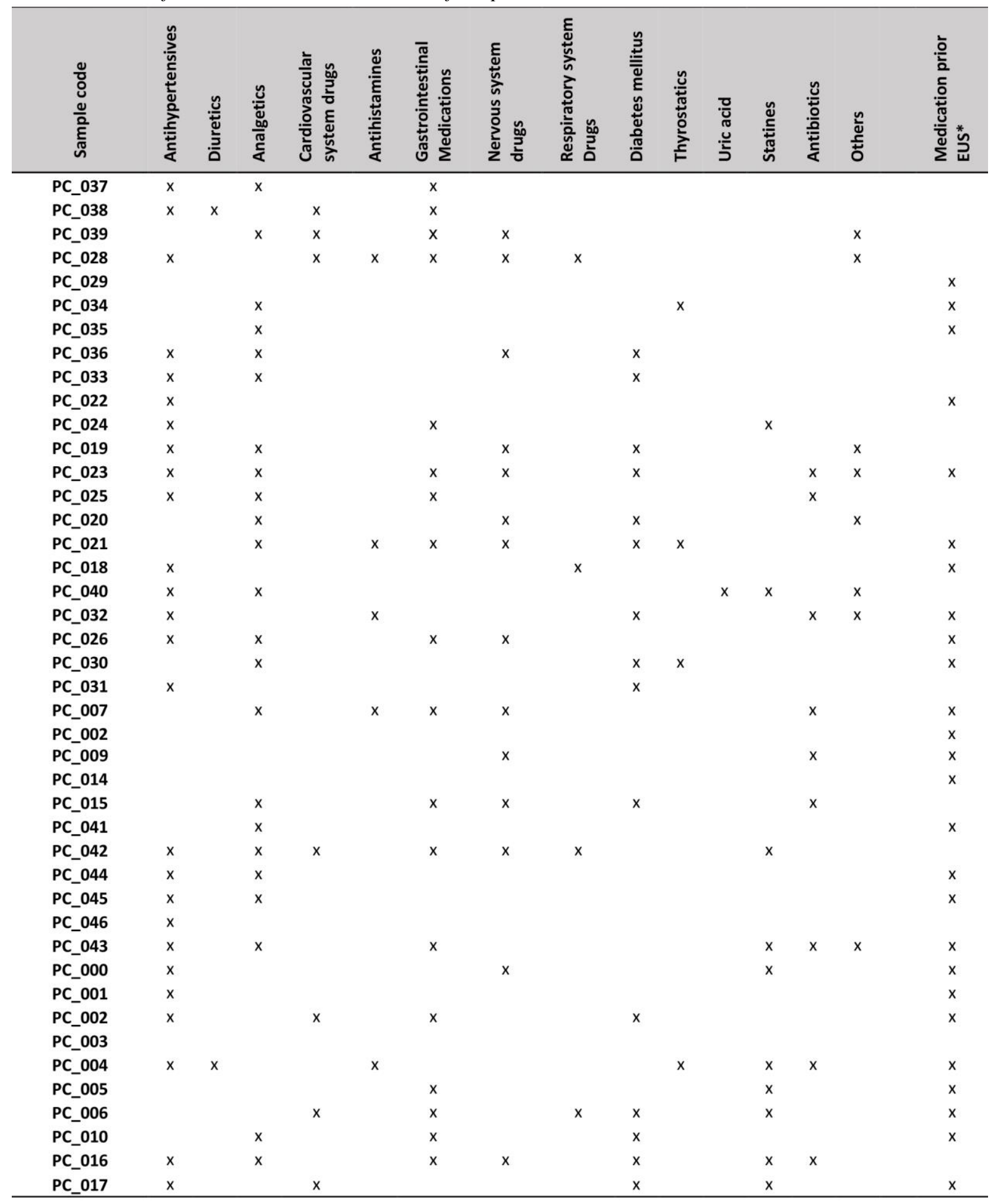


Table S3. A list of acute and chronic medication of T2DM patients.

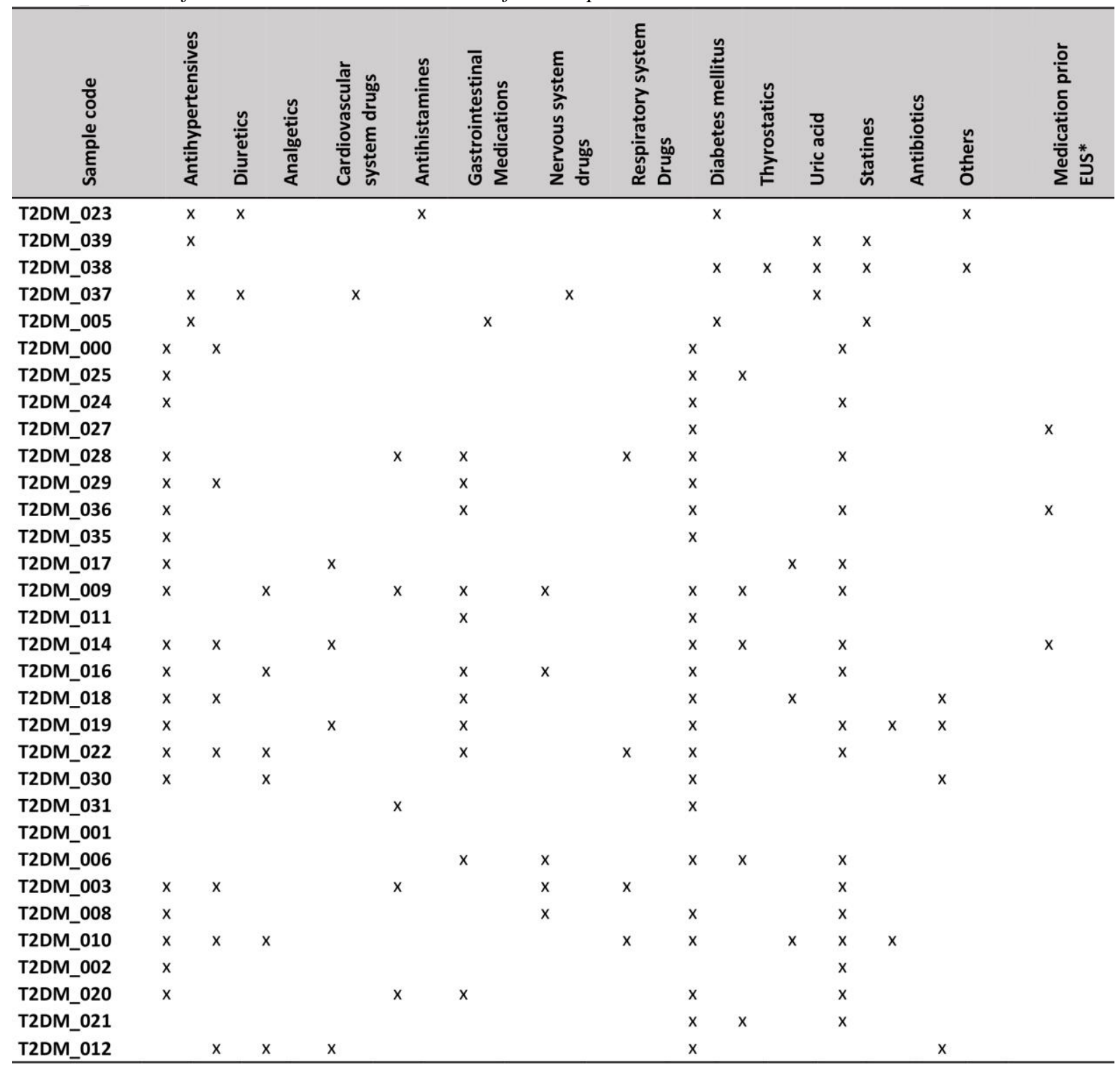




\section{Statistically significant metabolites}

Table S4. Statistically significant metabolites between pancreatic cancer patients (PC) and healthy controls (HC)

\begin{tabular}{l|l|l} 
Metabolite & $P$-value & $A U C$ \\
\hline Histidine* & $1.11 \mathrm{E}-08$ & 0.91339 \\
Mannose* & $6.58 \mathrm{E}-06$ & 0.83750 \\
Uridine* & $4.53 \mathrm{E}-06$ & 0.84375 \\
Creatine* & $1.05 \mathrm{E}-06$ & 0.86161 \\
Ornithine* & $1.05 \mathrm{E}-06$ & 0.86339 \\
Alanine* & $1.02 \mathrm{E}-06$ & 0.86875 \\
Serine* & $8.31 \mathrm{E}-05$ & 0.80089 \\
Lysine* & $3.89 \mathrm{E}-05$ & 0.81250 \\
Glutamine* & $3.60 \mathrm{E}-05$ & 0.81518 \\
Glycine* & $9.86 \mathrm{E}-04$ & 0.75714 \\
Threonine* & $6.86 \mathrm{E}-04$ & 0.76429 \\
3-Hydroxybutyrate* & $6.86 \mathrm{E}-04$ & 0.76429 \\
Carnitine* & $2.40 \mathrm{E}-04$ & 0.78304 \\
Methionine* & $1.25 \mathrm{E}-04$ & 0.79375 \\
Asparagine & $9.97 \mathrm{E}-03$ & 0.71071 \\
Tyrosine & $5.43 \mathrm{E}-03$ & 0.72411 \\
Arginine & $3.80 \mathrm{E}-03$ & 0.73214 \\
Proline & $2.73 \mathrm{E}-02$ & 0.67857 \\
Creatinine & $2.54 \mathrm{E}-02$ & 0.68125 \\
2-Hydroxyisovalerate & $2.37 \mathrm{E}-02$ & 0.68393 \\
Pyruvate & $2.12 \mathrm{E}-02$ & 0.68839 \\
Isobutyrate & $2.12 \mathrm{E}-02$ & 0.68750 \\
Acetoacetate & $1.51 \mathrm{E}-02$ & 0.69732 \\
Methanol & $1.40 \mathrm{E}-02$ & 0.70089 \\
Glutamate & $1.40 \mathrm{E}-02$ & 0.70000 \\
Valine & $1.32 \mathrm{E}-02$ & 0.70357 \\
\hline & &
\end{tabular}


Table S5. Statistically significant metabolites between long-term type 2 diabetes mellitus patients (T2DM) and healthy controls (HC)

\begin{tabular}{l|l|l} 
Metabolite & \multicolumn{1}{l}{$P$-value } & \multicolumn{1}{l}{$A U C$} \\
\hline Ornithine* & $9.96 \mathrm{E}-05$ & 0.83525 \\
Histidine* & $1.35 \mathrm{E}-05$ & 0.86751 \\
Glutamine* & $4.28 \mathrm{E}-04$ & 0.8053 \\
Uridine* & $2.59 \mathrm{E}-04$ & 0.81682 \\
Threonine & $7.22 \mathrm{E}-03$ & 0.74078 \\
Serine & $4.73 \mathrm{E}-03$ & 0.75115 \\
Tryptophan & $3.46 \mathrm{E}-03$ & 0.76152 \\
Phenylalanine & $3.46 \mathrm{E}-03$ & 0.75922 \\
Arginine & $2.27 \mathrm{E}-03$ & 0.77189 \\
Carnitine & $2.23 \mathrm{E}-03$ & 0.77535 \\
Formate & $4.48 \mathrm{E}-02$ & 0.68433 \\
Methionine & $1.78 \mathrm{E}-02$ & 0.70853 \\
Betaine & $1.78 \mathrm{E}-02$ & 0.70853 \\
Glucose & $1.73 \mathrm{E}-02$ & 0.71313 \\
Isopropanol & $1.70 \mathrm{E}-02$ & 0.71544 \\
Glycine & $1.73 \mathrm{E}-02$ & 0.71198 \\
Lysine & $1.65 \mathrm{E}-02$ & 0.71774 \\
Tyrosine & $1.61 \mathrm{E}-02$ & 0.72005 \\
Succinate & $1.50 \mathrm{E}-02$ & 0.7235 \\
\hline
\end{tabular}

Table S6. Statistically significant metabolites between pancreatic cancer patients (PC) and long-term type 2 diabetes mellitus patients (T2DM)

\begin{tabular}{l|l|l} 
Metabolite & $P$-value & $A U C$ \\
\hline Creatine* & $3.87 \mathrm{E}-05$ & 0.82903 \\
Mannose* & $4.05 \mathrm{E}-04$ & 0.78629 \\
Alanine* & $1.48 \mathrm{E}-04$ & 0.80403 \\
Valine & $4.10 \mathrm{E}-02$ & 0.68387 \\
Tryptophan & $4.05 \mathrm{E}-02$ & 0.68548 \\
Lysine & $3.74 \mathrm{E}-02$ & 0.69032 \\
Propionate & $3.63 \mathrm{E}-02$ & 0.69516 \\
Propylene glycol & $3.63 \mathrm{E}-02$ & 0.69355 \\
Glutamate & $3.63 \mathrm{E}-02$ & 0.68387 \\
3-Hydroxybutyrate & $3.37 \mathrm{E}-02$ & 0.70484 \\
Proline & $1.41 \mathrm{E}-02$ & 0.72581 \\
\hline
\end{tabular}

Table S7. Confusion matrix for simultaneous discrimination of PC, T2DM and HC.

\begin{tabular}{|c|c|c|c|}
\hline & PC & T2DM & HC \\
\hline PC & 79.4 & 11.7 & 8.9 \\
\hline T2DM & 10.1 & 74.6 & 15.3 \\
\hline HC & 9.7 & 8.3 & 82.0 \\
\hline
\end{tabular}


Metabolic pathway analysis

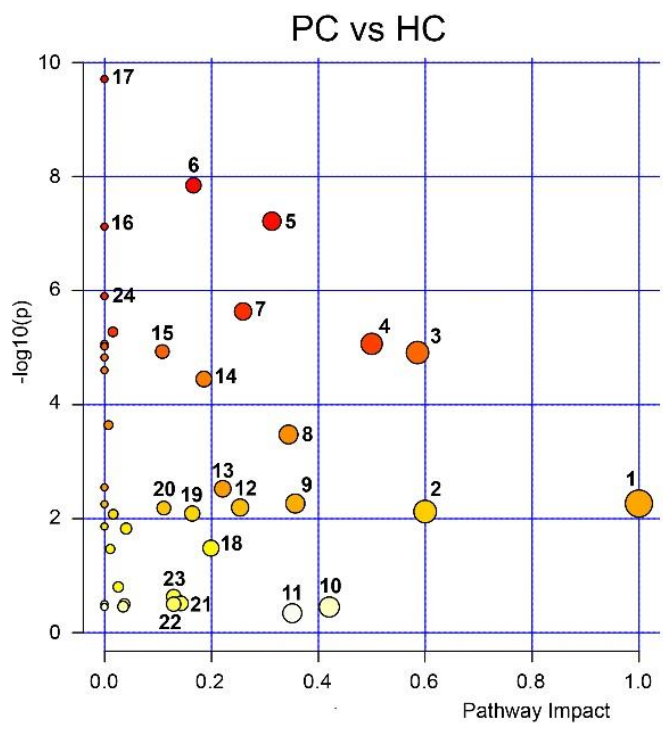

1) Phenylalanine, tyrosine and tryptophan biosynthesis

2) Synthesis and degradation of ketone bodies

3) Glycine, serine and threonine metabolism

4) D-glutamine and D-glutamate metabolism

5) Alanine, aspartate and glutamate metabolism

6) Aminoacyl-tRNA biosynthesis

7) Glyoxylate and dicarboxylate metabolism

8) Arginine and proline metabolism

9) Phenylalanine metabolism

10) Starch and sucrose metabolism

11) Pyruvate metabolism

12) Arginine biosynthesis

13) Histidine metabolism

14) Cysteine and methionine metabolism

15) Glutathione metabolism

16) Selenocompound metabolism

17) $\beta$-alanine metabolism

18) Citrate cycle (TCA cycle)

19) Tyrosine metabolism

20) Butanoate metabolism

21) Tryptophan metabolism

22) Inositol phosphate metabolism

T2DM vs HC

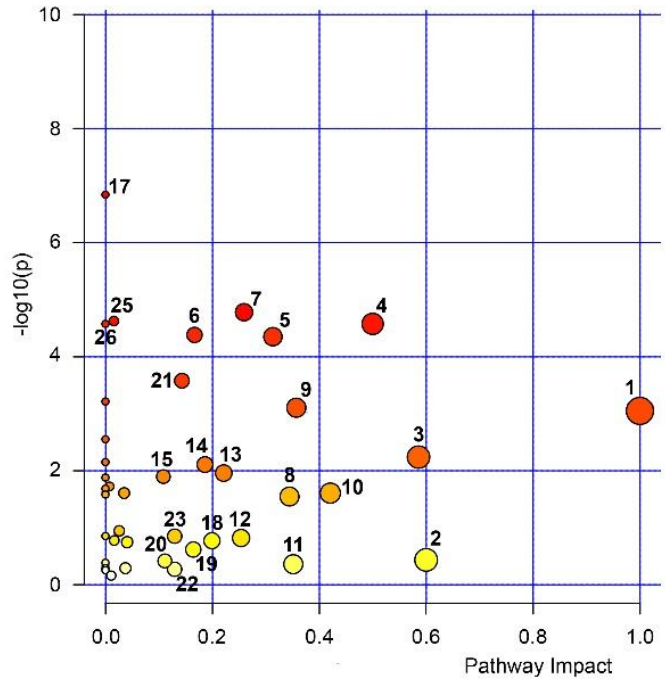

23) Glycolysis/Gluconeogenesis

24) Amino sugar and nucleotide sugar metabolism

25) Pyrimidine metabolism

26) Nitrogen metabolism

27) Porphyrin and chlorophyll metabolism

28) Biotin metabolism

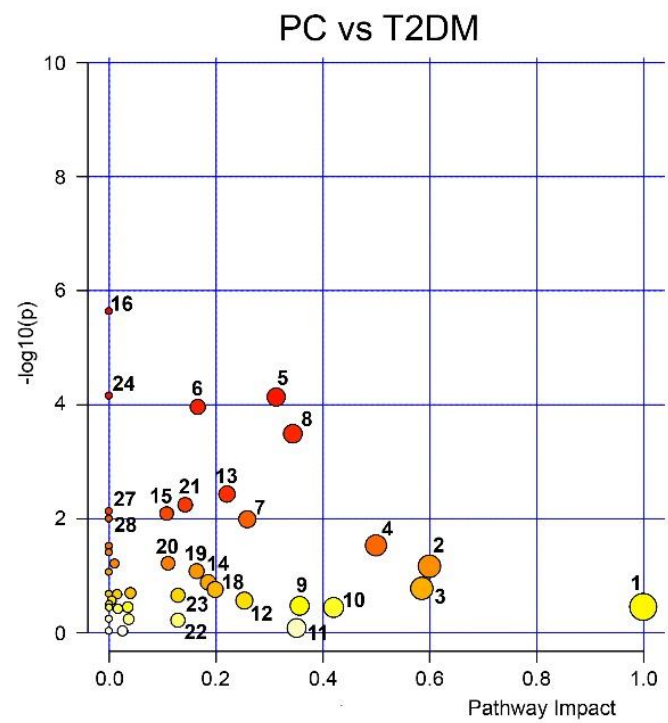

Figure S1. Metabolic pathway analysis of the group discrimination. Pancreatic cancer patients (PC), long-term type 2 diabetes mellitus patients (T2DM) and healthy controls (HC). 
Principal component analysis on concentration data
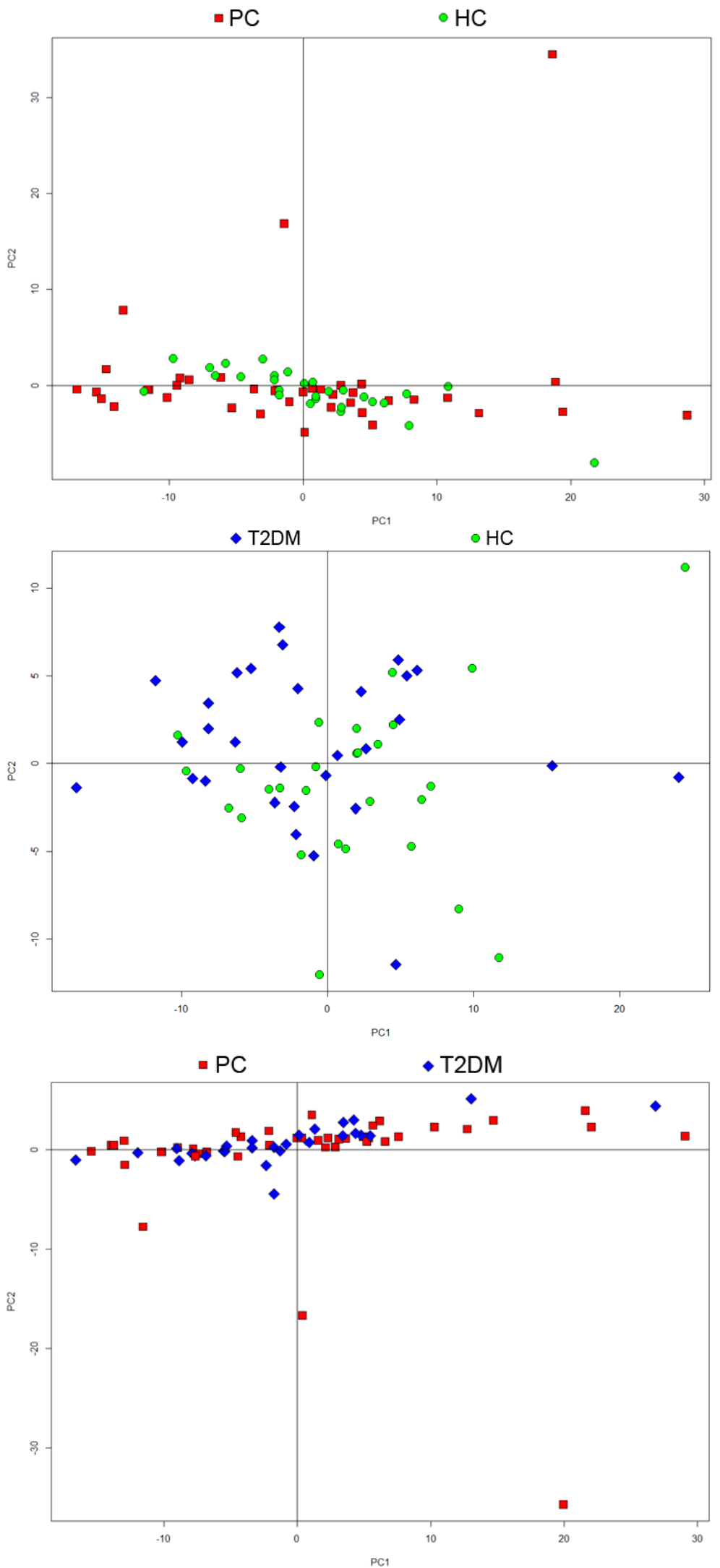

Figure S2. Principal component analyses of group differences based on concentration data. Pancreatic cancer patients (PC), long-term type 2 diabetes mellitus patients (T2DM) and healthy controls (HC). 


\section{Discriminant analysis of binning data}

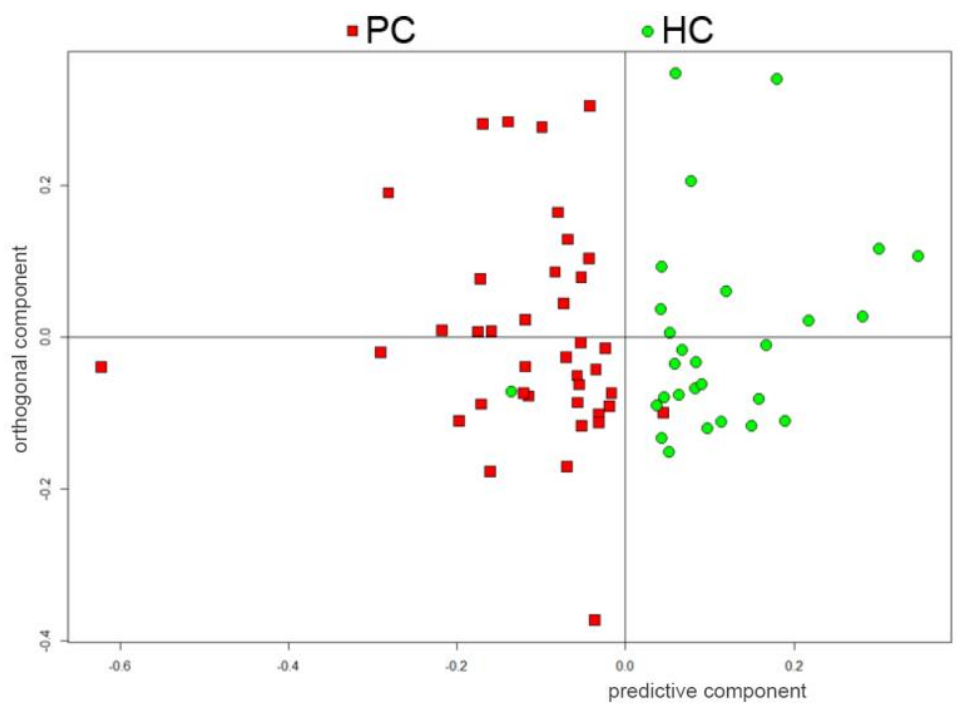

Confusion matrix
\begin{tabular}{|c|c|c|}
\hline & PC & HC \\
\hline PC & 87.3 & 12.7 \\
\hline HC & 7.7 & 92.3 \\
\hline
\end{tabular}

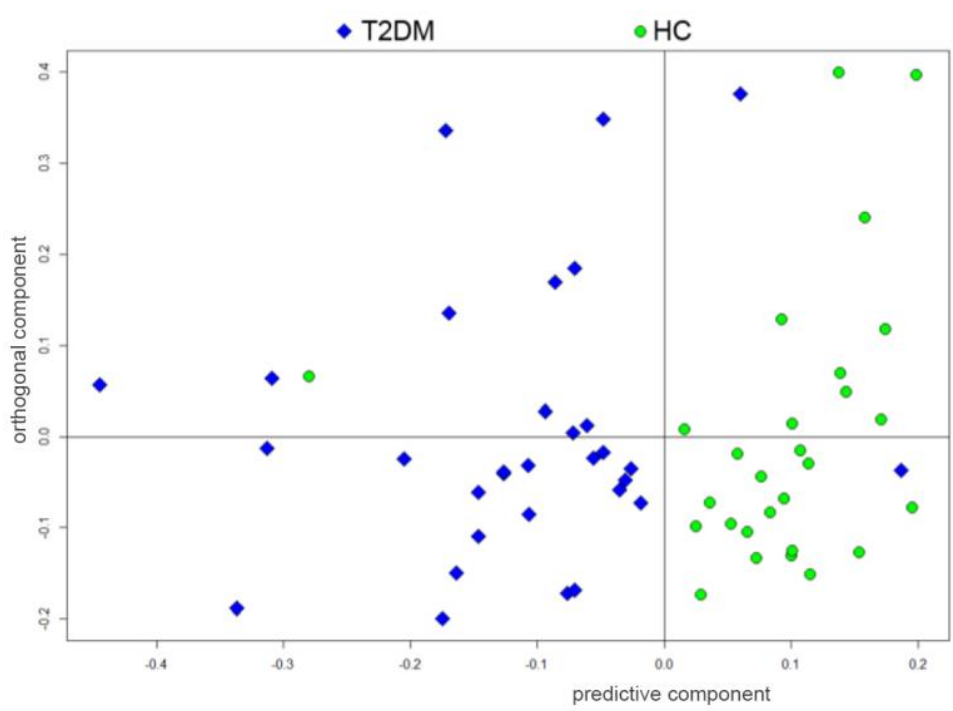

Confusion matrix
\begin{tabular}{|c|c|c|}
\hline & T2DM & HC \\
\hline T2DM & 79.6 & 20.4 \\
\hline HC & 11.3 & 88.7 \\
\hline
\end{tabular}

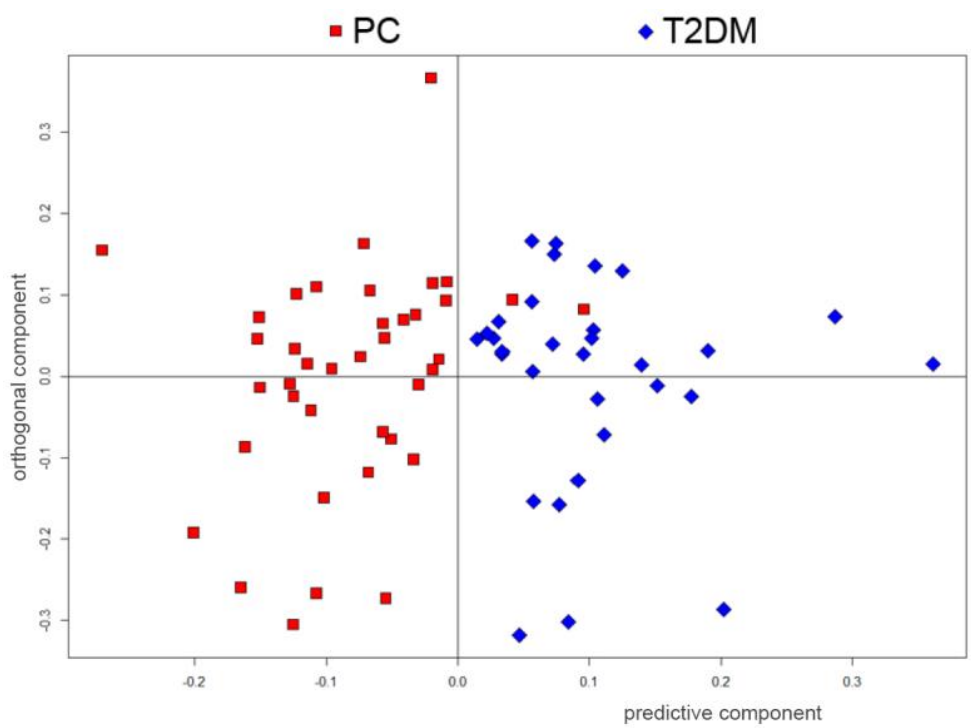

Confusion matrix
\begin{tabular}{|c|c|c|}
\hline & PC & T2DM \\
\hline PC & 81.9 & 18.1 \\
\hline T2DM & 14.0 & 86.0 \\
\hline
\end{tabular}

Figure S3. OPLS-DA based on binning data. Pancreatic cancer patients (PC), long-term type 2 diabetes mellitus patients (T2DM) and healthy controls (HC). 


\section{Univariate statistical analyses}
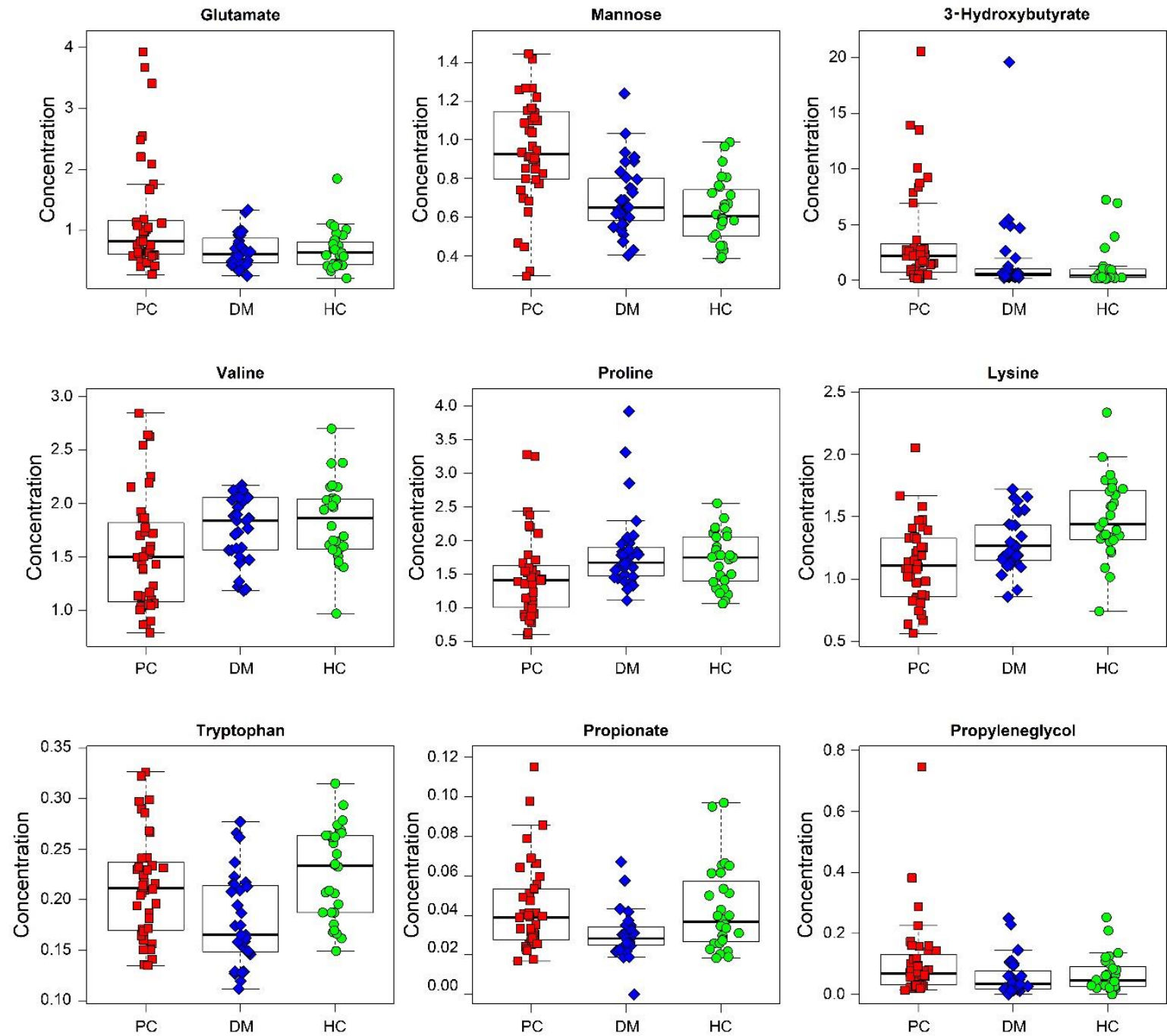

Figure S4. Box plots of dysregulated metabolites between pancreatic cancer patients (PC), long-term type 2 diabetes mellitus patients (T2DM) and healthy controls (HC). 


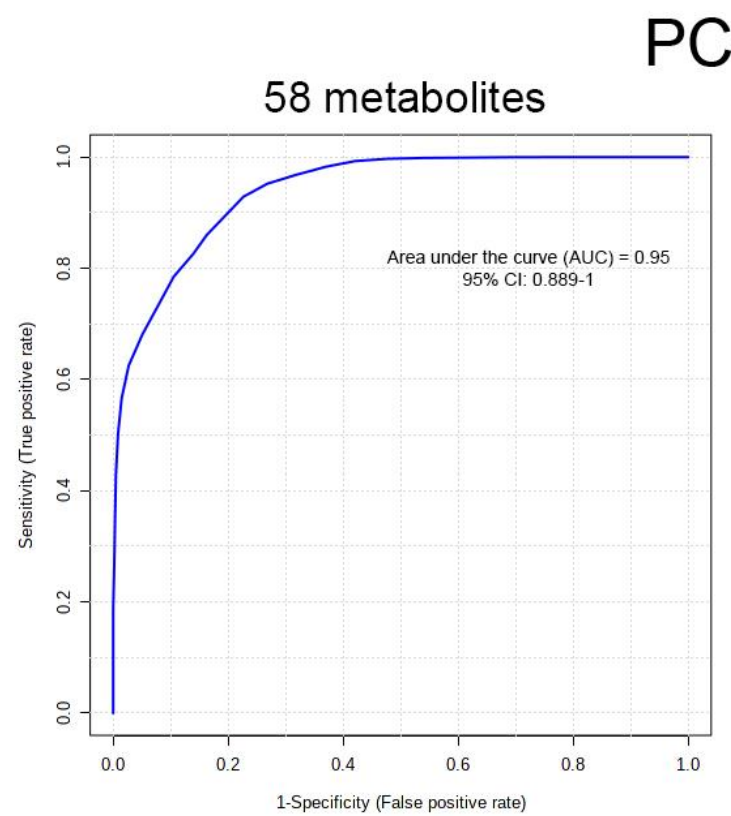

\section{vs $\mathrm{HC}$}
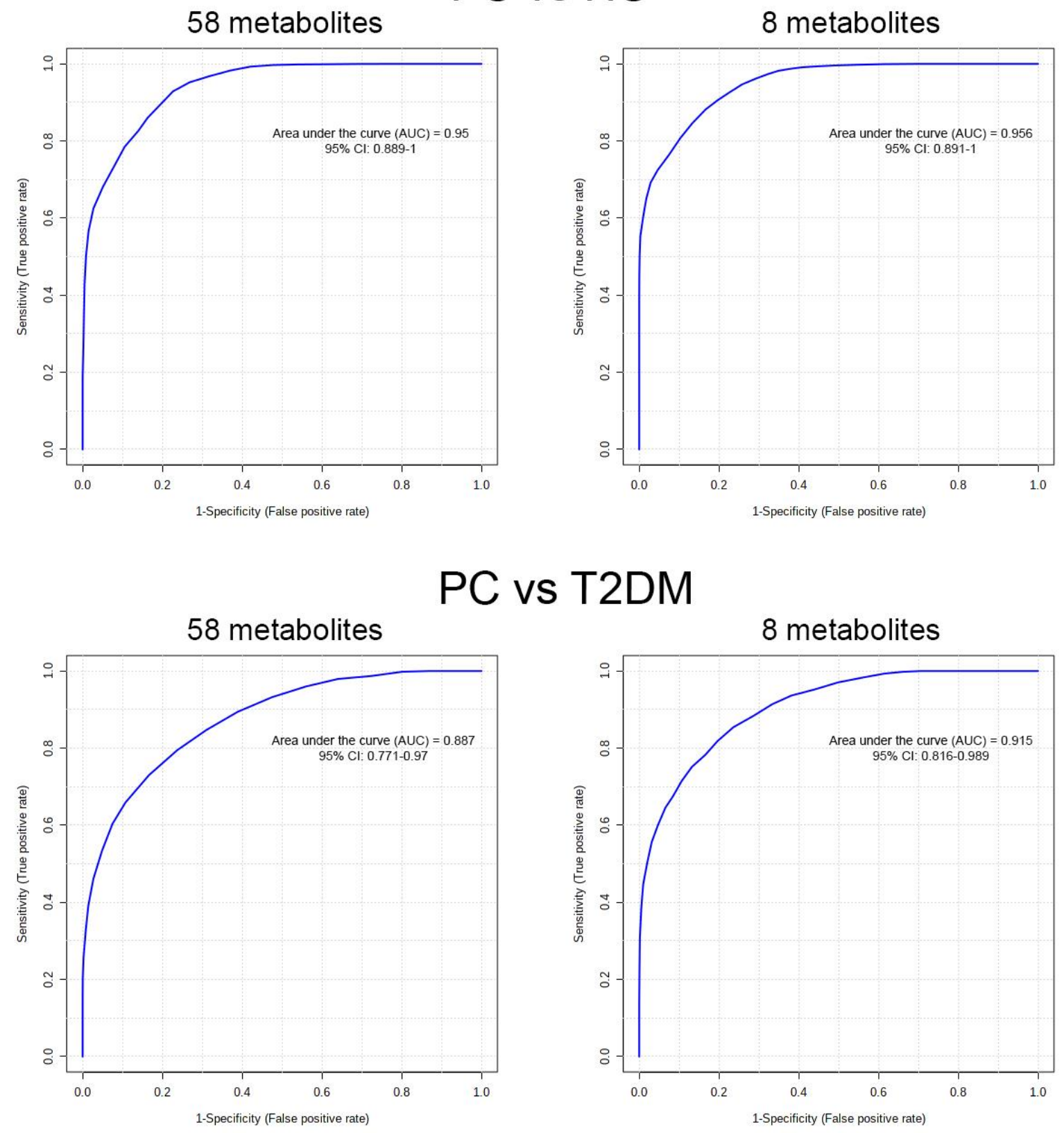

Figure S5. ROC curves using all 58 metabolites and for specific 8-metabolite panel discriminating between pancreatic cancer patients (PC) and healthy controls (HC) and pancreatic cancer patients (PC) and long-term type 2 diabetes mellitus patients (T2DM). 


\section{Clinical examination of at-risk individuals}

Patient RODM 003 has no other health issues except for the recently diagnosed diabetes mellitus, which was diagnosed in December 2015; Metformin treatment was deployed. EUS is without any signs of pathology; a control EUS is scheduled within one year.

Patient RODM 008 suffered from severe chronic pancreatitis accompanied by pancreatic duct stenosis in the neck, pancreaticolithiasis and intraductal papillary mucinous neoplasm (IPMN) side branch cysts in the head of the pancreas. A stenosis resection was proposed; however, the patient refused. A recent $\mathrm{CT}$ and MR are without signs of malignant transformation (Figure S6). EUS-FNA of the pancreatic duct stenosis area was negative. Diabetes was diagnosed in January 2016 randomly as a part of a preoperative gynecological examination.

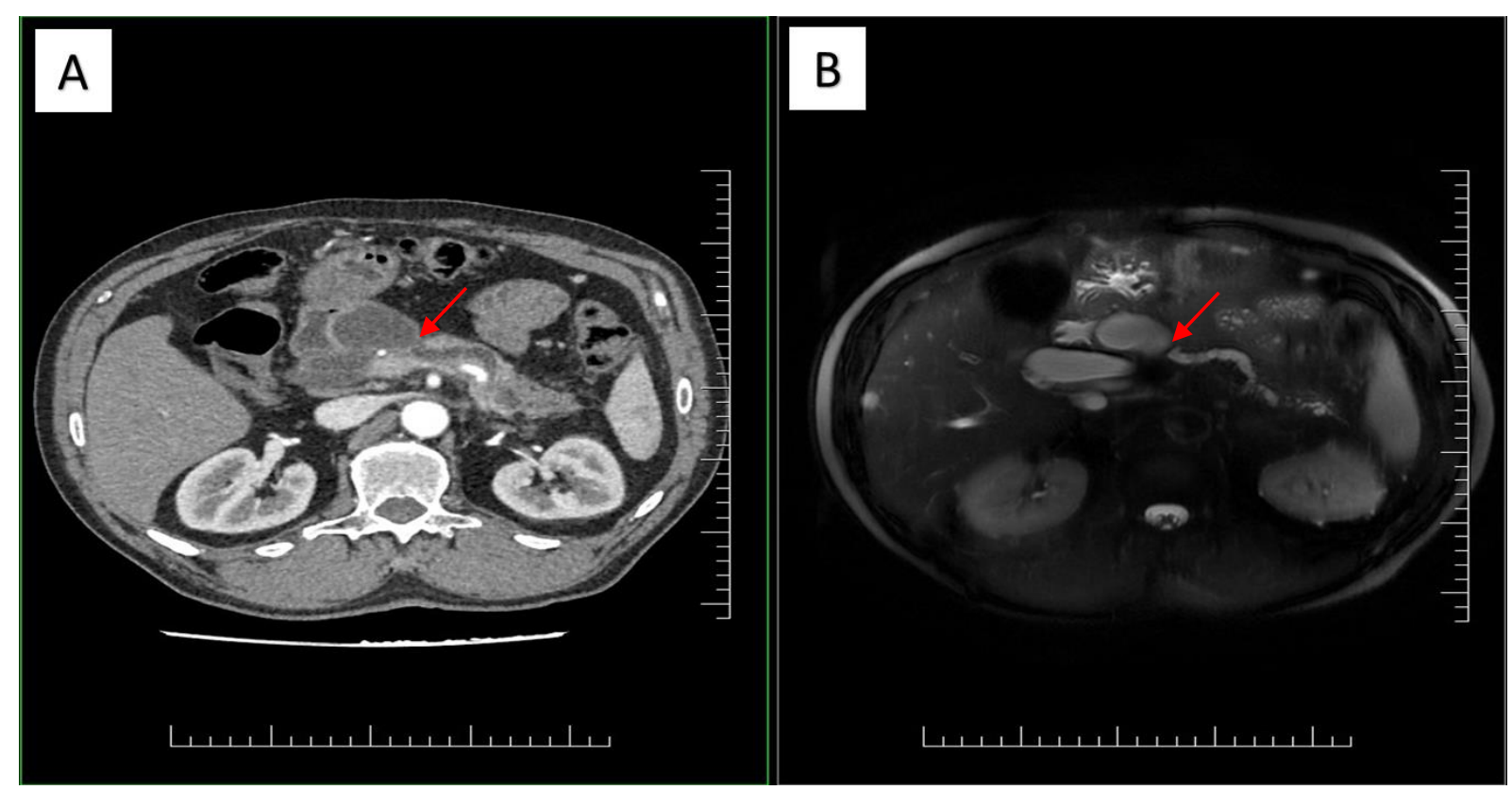

Figure S6. CT (A) and MR (B) showing a pancreatic cyst in the head causing the stenosis of pancreatic duct marked with an arrow (RODM 008).

Patient RODM 011 suffered from acute pancreatitis in 2015. At the same time, he was diagnosed with diabetes mellitus. According to the CT, MRI and EUS performed in 2016, a dilatation of branch pancreatic ducts was found in the pancreatic neck. These findings were primarily attributed to the IPMN without high-risk features. In 2017 during routine follow-up, the patient underwent MRI, which revealed a short pancreatic duct stenosis (Figure S7). EUS-FNA of this lesion proved a suspicious dysplastic tissue. At this time, blood collection for the metabolomic analysis was performed. The patient was indicated for pancreatic head and neck surgery in early 2017. The final histology proved IPMN with poorly differentiated pancreatic cancer. 

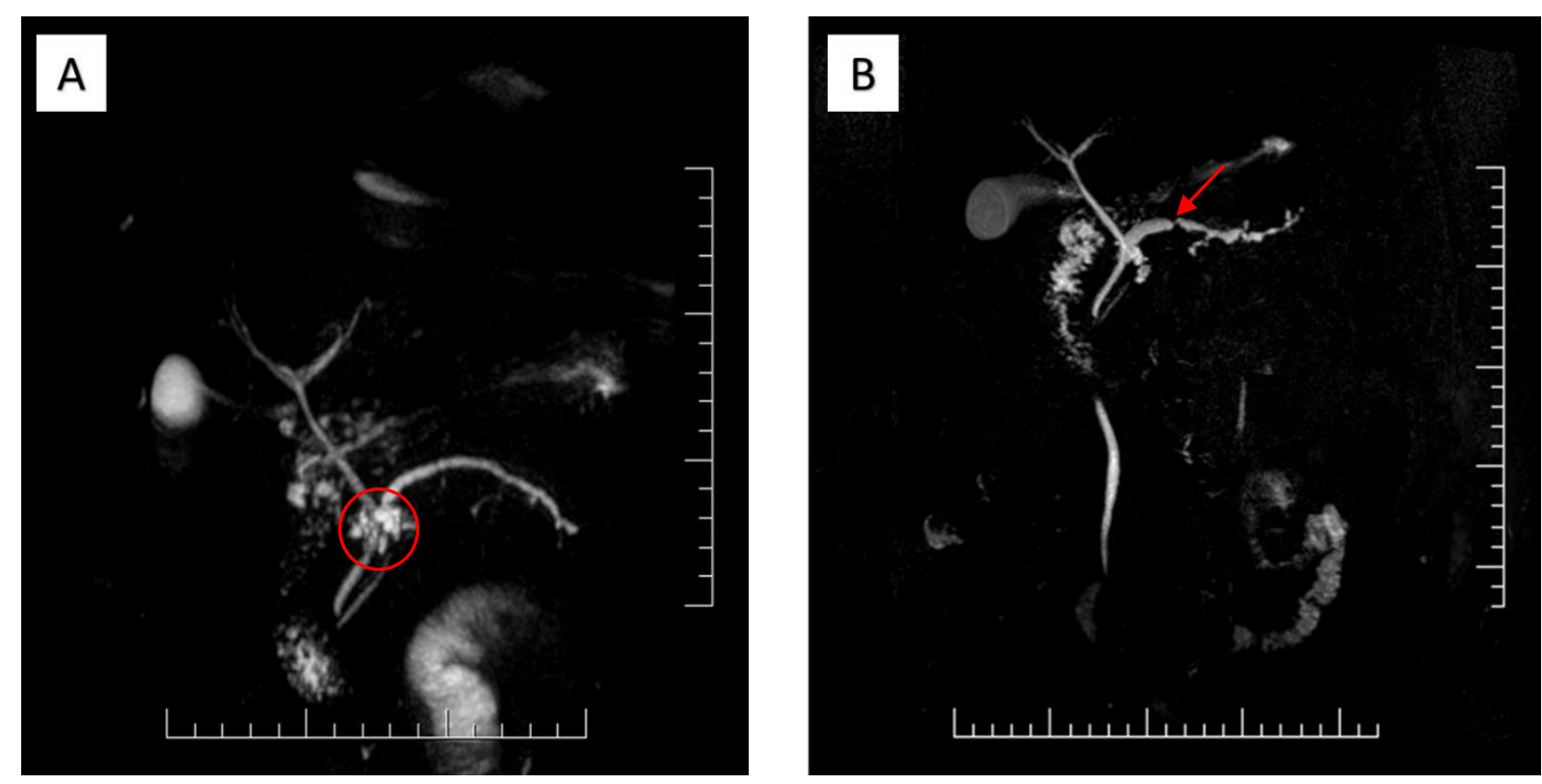

Figure S7. (A) MR showing cystic lesion of pancreatic neck (marked with a circle); (B) MR folow up with cystic lesion of pancreatic neck and newly detected stenosis of pancreatic duct (marked with an arrow) (RODM 011).

Patient RODM 015 is under regular follow-up because of a family history of PC. Blood sample collection was made in April 2017. Diabetes was diagnosed based on the result obtained. EUS showed cysts in the pancreatic body. Recently, a new cyst with a hypoechogenic area of around $15 \mathrm{~mm}$ was localized in the pancreatic tail (Figure S8). Currently, EUS-FNA is scheduled. Based on the histology, the pancreatic tail resection will be discussed by the Multidisciplinary Pancreatic Board of the Military University Hospital Prague.
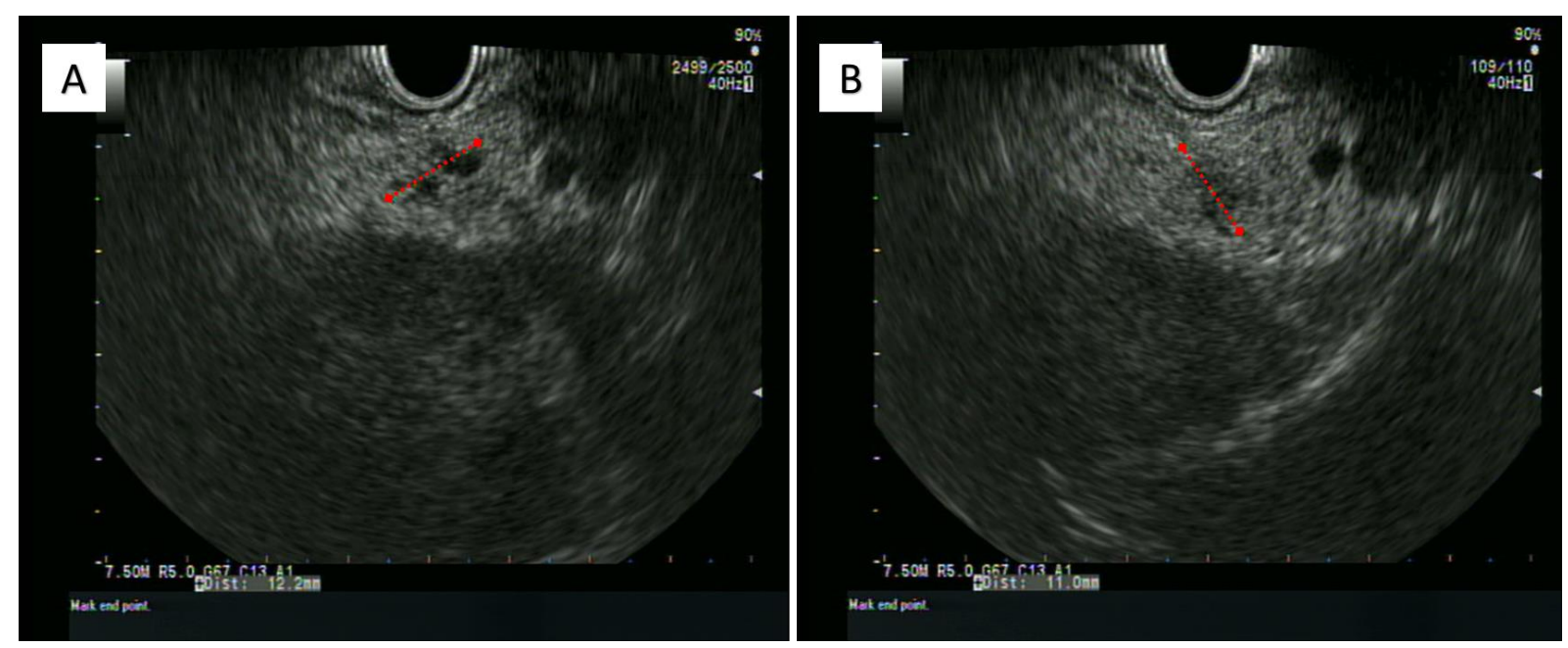

Figure S8. (A) EUS with small cystic lesion in pancreatic tail; (B) EUS with hypoechogenic area around pancreatic duct (RODM 015).

Patient RODM 036 was originally diagnosed with cholelithiasis. Blood sample collection for this study was made in June 2017. Diagnosis of diabetes was made in August 2015. The patient underwent an extraction of a common bile duct stone by ERCP and a stone extraction by choledochotomy. Recently, the patient was diagnosed with repeated pancreatitis. CT revealed a cystic lesion with dilatation of 
pancreatic duct accompanied by a stenosis of common bile duct, which led to indication for resection (Figure S9). The patient underwent a pancreatoduodenectomy, and the resection specimen showed advanced chronic inflammatory changes with numerous necrosis of the acinar tissue.

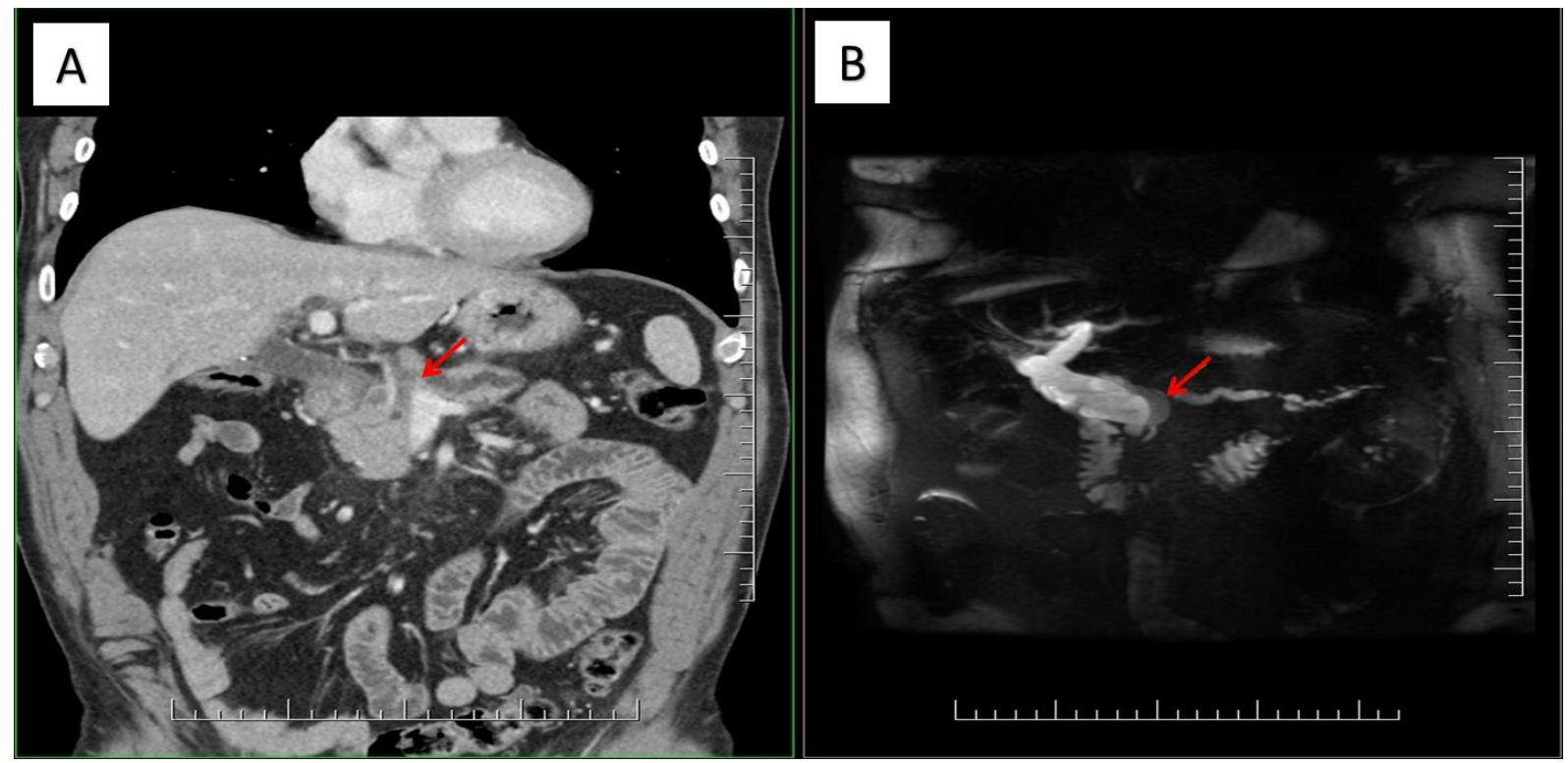

Figure S9. CT (A) and MR (B) showing discontinuity and dilatation of panctreatic duct (marked with an arrow) (RODM 036).

Patient RODM 071 suffered from chronic cholecystitis. Blood sample collection was made in March 2019. Diabetes was diagnosed based on the result obtained. The patient appeared without serious problems after an established diet, except for unexplained epigastric pain with propagation to the back. MR and CT is depicted in Figure S10. Pancreatic lipomatosis was described on abdominal ultrasound; EUS is scheduled.
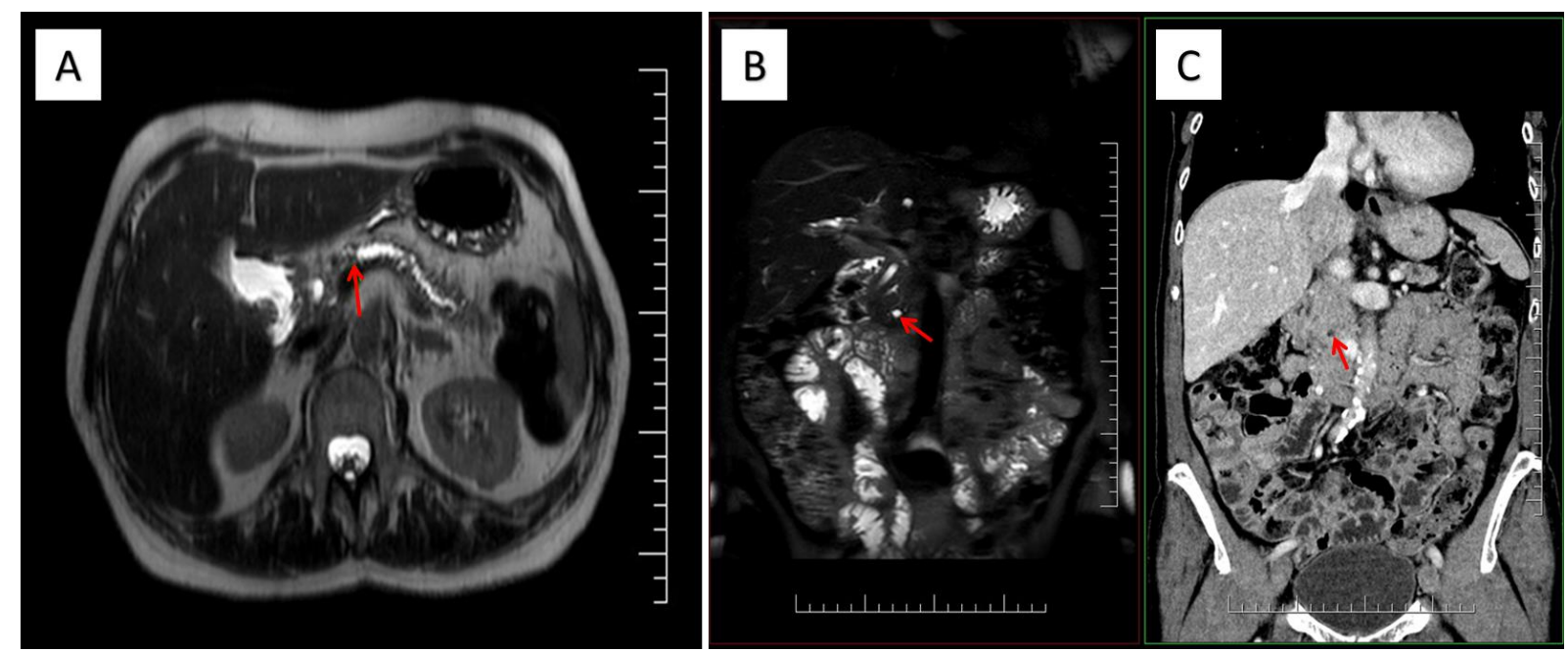

Figure S10. (A) MR with a stenosis of pancreatic duct (marked with an arrow); (B) MR and (C) CT with small branch duct IPMN in the pancreatic head (marked with an arrow) (RODM 071). 